\title{
Efficacy of a small-caliber colonoscope for pain in female patients during unsedated colonoscopy: a randomized controlled study
}

\section{다 $(9) \Theta$}

\author{
Authors \\ Yasuhiko Hamada ${ }^{1}$, Kyosuke Tanaka², Masaki Katsurahara² ${ }^{2}$, Noriyuki Horiki², Reiko Yamada ${ }^{1}$, Junya Tsuboi ${ }^{2}$, Misaki \\ Nakamura', Satoshi Tamaru ${ }^{3}$, Tomomi Yamada ${ }^{4}$, Yoshiyuki Takei ${ }^{1}$
}

Institutions

1 Department of Gastroenterology and Hepatology, Mie University Hospital, Tsu, Japan

2 Department of Endoscopy, Mie University Hospital, Tsu, Japan

3 Clinical Research Support Center, Mie University Hospital, Tsu, Japan

4 Department of Medical Innovation, Osaka University Hospital, Suita, Japan

submitted 25.9.2020

accepted after revision 2.3.2021

\section{Bibliography}

Endosc Int Open 2021; 09: E1055-E1061

DOI 10.1055/a-1464-0780

ISSN 2364-3722

(C) 2021. The Author(s).

This is an open access article published by Thieme under the terms of the Creative Commons Attribution-NonDerivative-NonCommercial License, permitting copying and reproduction so long as the original work is given appropriate credit. Contents may not be used for commercial purposes, or adapted, remixed, transformed or built upon. (https://creativecommons.org/licenses/by-nc-nd/4.0/)

Georg Thieme Verlag KG, Rüdigerstraße 14,

70469 Stuttgart, Germany

Corresponding author

Yasuhiko Hamada, MD, PhD, Department of Gastroenterology and Hepatology, Mie University Hospital, 2-174 Edobashi, Tsu, Mie, 514-8507, Japan

Fax: +81-59-231-5200

y-hamada@clin.medic.mie-u.ac.jp

\section{ABSTRACT}

Background and study aims Female sex has been identified as a factor increasing patients' pain during colonoscopy. The aim of this randomized controlled study was to investigate the efficacy of a small-caliber colonoscope, PCFPQ260 L, for limiting pain in women during unsedated colonoscopy.

Patients and methods Women who underwent unsedated colonoscopy were randomly allocated to either the small-caliber or standard colonoscope group. The primary outcome was overall pain and secondary outcomes were maximum pain and procedural measures. In addition, the effects of colonoscope type were analysed using analysis of covariance and logistic regression with adjustment for stratification factors, age and prior abdomino-pelvic surgery.

Results A total of 220 women were randomly assigned to the small-caliber $(n=110)$ or standard $(n=110)$ colonoscope groups. Overall and maximum pain scores were significantly lower in the small-caliber colonoscope group than the standard colonoscope group (overall pain, 20.0 vs. $32.4, \mathrm{P}<0.0001$; maximum pain, 28.9 vs. $47.2, \mathrm{P}<$ $0.0001)$. The small-caliber colonoscope group achieved a superior cecal intubation rate ( $99 \%$ vs. $93 \%, \mathrm{P}=0.035)$. The rate of patient acceptance of unsedated colonoscopy in the future was higher in the small-caliber colonoscope group than in the standard colonoscope group ( $98 \%$ vs. $87 \%, \mathrm{P}=0.003)$. In addition, the small-caliber colonoscope was superior with respect to reducing pain and improving the rate of patient acceptance of unsedated colonoscopy with adjustment.

Conclusions This study demonstrates the efficacy of the small-caliber colonoscope for reducing pain in women and improving their rate of acceptance of unsedated colonoscopy.

\section{Introduction}

Colorectal cancer (CRC) is one of the most common cancers and cause of cancer deaths worldwide [1]. Colonoscopy is the standard modality for CRC screening and surveillance, and has significantly reduced the mortality of CRC [2]. However, patient pain arising from colonoscopy is one of the most important factors that discourages individuals from participating in CRC screening and surveillance [3]. Thus, a number of techniques 
and equipment modifications have been investigated to reduce patient pain during colonoscopy, including water infusion methods [4], attaching a transparent hood to the colonoscope tip [5], magnetic endoscopic imaging [6], colonoscopes with variable stiffness features [7], upper gastrointestinal endoscopes [8], and small-caliber colonoscopes [9].

Female sex has been reported to be a predictor of longer intubation time, regardless of the endoscopist's experience, because the female colon is sometimes angulated and with adhesions due to previous gynecological surgery $[10,11]$. Accordingly, excessive looping is more frequent in women and causes more severe pain than in men during colonoscopy [12]. As a result, women are more likely than men to avoid colonoscopy. Although sedation can help to decrease the pain in women during colonoscopy [13], overuse of sedation may increase the cost of medical care, sedation-related adverse events (AEs), and procedure-related AEs $[14,15]$. Thus, painless colonoscopy without sedation can overcome these disadvantages and is considered the optimal colonoscopy for women.

Several randomized controlled trials (RCTs) have shown that the use of a small-caliber colonoscope PCF-PQ260 L (Olympus Medical Systems, Tokyo, Japan) was associated with reduced pain during colonoscopy, compared to a standard colonoscope [9, 16-19]. This small-caliber colonoscope has an outer diameter of $9.2 \mathrm{~mm}$ and two additional mechanisms, "passive bending" and "high force transmission" [20]. Passive bending improves insertion through angulated or stenotic segments of the colon. High force transmission improves the control of force and torque. Although studies comparing the PCF-PQ260 $L$ to standard colonoscopes have been reported, the value of this small-caliber colonoscope for reducing patient pain, focusing on female patients and especially without sedation, has not been investigated completely. Thus, we conducted a RCT to investigate the efficacy of the small-caliber colonoscope for pain in women during unsedated colonoscopy.

\section{Patients and methods}

\section{Study overview}

We conducted a single-blinded (only the subjects were blinded), randomized controlled study between October 2013 and December 2017 at Mie University Hospital. The subjects in this study underwent colonoscopy using either a small-caliber colonoscope or a standard colonoscope. The study protocol was approved by the Clinical Research Ethics Review Committee of Mie University Hospital on April 13, 2013 (approval number, 2511). The study was registered in the University Hospital Medical Network Clinical Trials Registry (UMIN-CTR; www.umin.ac.jp/ ctr/index/htm) as ID number UMIN000010350 on April 1, 2013. The study was conducted in accordance with the approved protocol and ethical guidelines of the Declaration of Helsinki.

\section{Study population}

Women who were scheduled to undergo total colonoscopy were considered eligible for inclusion in this study. The exclusion criteria were defined as follows: (1) age < 20 years; (2) his-

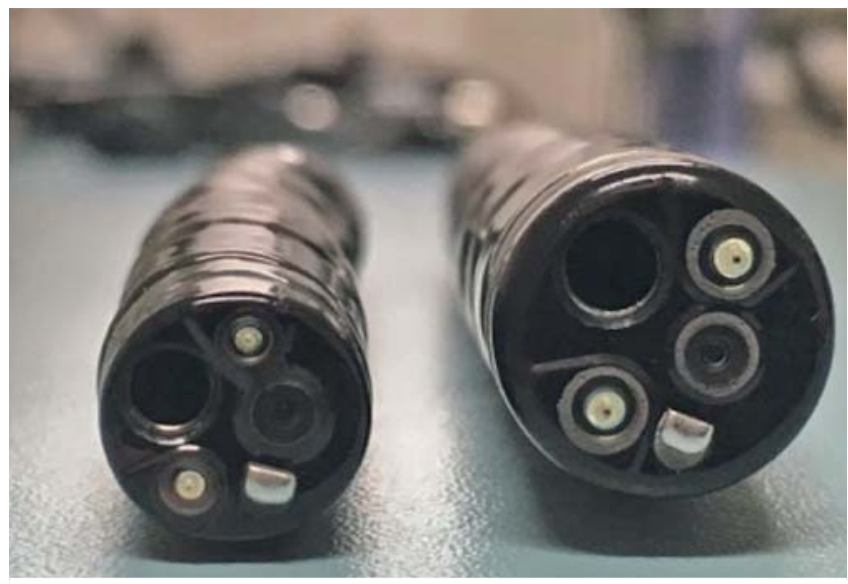

- Fig. 1 Features of the small-caliber colonoscope (9.2-mm diameter, PCF-PQ260 L, left) and the standard colonoscope (12.2-mm diameter, CF-Q260Al, right).

tory of colorectal surgical resection, (3) pregnancy; (4) inflammatory bowel disease; (5) massive hematochezia; and (6) preference for undergoing sedation. Written informed consent was obtained from all patients before enrollment.

\section{Randomization}

Patients were randomized using a computer-generated system from the Mie University Hospital Clinical Research Support Center. Using a minimization algorithm, the colonoscope selection was balanced with respect to two stratification variables: age ( $<60$ and $\geq 60$ years) and prior abdominopelvic surgery (presence and absence). The website was only available to the trial investigators. Patients, but not the endoscopist and medical assistant, were blinded to the colonoscope used.

\section{Colonoscope}

We used a PCF-PQ260L for the small-caliber colonoscope group and a CF-Q260AI (Olympus Medical Systems, Tokyo, Japan) for the standard colonoscope group. Features of these colonoscopes are shown in > Fig. 1 (left, PCF-PQ260L; right, CFQ260Al). The small-caliber colonoscope has an outer diameter of $9.2 \mathrm{~mm}$ and a working length of $168 \mathrm{~cm}$. In addition, the small-caliber colonoscope has a flexible passive bending section located close to the primary bending section at the distal side of the endoscope and a high-force transmission insertion tube for efficiently transmitting the force from the proximal to the distal side [20]. However, the variable-stiffness feature was not attached to the small-caliber colonoscope. On the other hand, the standard colonoscope has an outer diameter of $12.2 \mathrm{~mm}$, a working length of $133 \mathrm{~cm}$, and a variable stiffness feature. During colonoscopy, a 2-mm transparent cap was attached to the tip of the each colonoscope (distal attachment, small-caliber colonoscope; model MAJ-1988, standard colonoscope; model MAJ-1990, Olympus Medical Systems, Tokyo, Japan). 


\section{Bowel preparation and intervention procedures}

All patients underwent bowel preparation with sennoside calcium and sodium picosulfate hydrate the day before the examination and $2 \mathrm{~L}$ of polyethylene glycol solution during the morning of the examination. The endoscopists assessed the quality of bowel preparation according to the extent of mucosal visualization after suction of the fluid residue, using the Aronchick Bowel Preparation scale: excellent ( $\geq 95 \%$ mucosal visualization); good ( $90 \%$ to $95 \%$ mucosal visualization); fair ( $80 \%$ to $90 \%$ mucosal visualization); and poor (<80\% mucosal visualization) [21].

All procedures were performed by one of eight experienced (board-certified) endoscopists (Y. Hamada, K. Tanaka, M. Katsurahara, M. Nakamura, R. Yamada, T. Kitade, S. Tano, J. Tsuboi), each of whom had performed more than 2000 colonoscopies, and four inexperienced (non board-certified) endoscopists (T. Harada, T. Sakuno, H. Miura, H. Okuse), each of whom had performed fewer than 500 colonoscopies. At the time of study enrollment, the previous medical history of each patient was investigated using a questionnaire. Other relevant demographic variables, such as height and body weight, were measured by a medical assistant. To prevent colonic wall spasms, scopolamine butylbromide $(20 \mathrm{mg})$ or glucagon $(1 \mathrm{mg})$ was administered by intramuscular injection. Initially at least, patients did not receive any sedation. If the patient complained of severe pain or discomfort during colonoscopy, sedation was permitted at endoscopist discretion.

All patients underwent examination using air insufflation. Conventional insertion techniques, including loop resolution, position change, and abdominal compression were used if necessary. Cecal intubation was confirmed either by ileal intubation or visualization of both the appendix orifice and ileocecal valve. When the colonoscope could not be inserted further, the colonoscopy was unsuccessful and a second colonoscopy using the alternative colonoscope was performed in the same session by the same endoscopists (e. g. for a failed standard colonoscope procedure, the small-caliber colonoscope was used and vice versa). Withdrawal time was calculated by subtracting the cecal intubation time from the total procedure time.

Patient pain during colonoscopy was assessed using a $100-\mathrm{mm}$ visual analogue scale (VAS) with a score of 0 indicating no pain and 100 indicating extreme pain. Before the procedure, the endoscopists or medical assistants explained the VAS scoring system to the patients. After the procedure, the patients were asked to report the overall pain and maximum pain during the colonoscopy, assessed using VAS. In addition, patient acceptance of unsedated colonoscopy in the future was assessed using a questionnaire, with the responses being acceptable or unacceptable.

\section{Outcome measures}

The primary outcome was overall patient pain, using the VAS during undsedated colonoscopy. Secondary outcomes were: (1) patient maximum pain using VAS; (2) cecal intubation rate; (3) cecal intubation time; (4) withdrawal time; (5) total procedure time; (6) adenoma detection rate; (7) rate of abdominal compression used during colonoscopy; (8) rate of patient acceptance of unsedated colonoscopy in the future; and (9) rate of AEs within 14 days of the colonoscopy, such as perforation and hemorrhage.

\section{Sample size calculation}

Student's $t$-test was used to calculate the sample size. From the report of a previous study, we assumed the mean VAS score for overall pain in the standard colonoscope group was 25 (standard deviation [SD], 20) and the mean VAS score for overall pain in the small-caliber colonoscope group was 17 (SD, 20) [18]. Thus, the small-caliber colonoscope group had a $32 \%$ lower mean VAS score for overall pain than the standard colonoscope group. Therefore, a minimum of 200 subjects would be required ( 100 patients each for group) to achieve a power of $80 \%$ with a significant level of 0.05 (2-sided). Assuming an approximate $10 \%$ deviation rate, we aimed to enroll 220 subjects for full assessment.

\section{Statistical analysis}

Intention-to-treat analysis was conducted for all comparisons of patients who were enrolled this study. Continuous variables were expressed as the mean with SD and compared using the Student's t-test. Categorical data were analyzed using the chisquared test when appropriate; otherwise, the Fisher exact test was used. $P<0.05$ was considered to be statistically significant and all tests were two-sided. The differences in effects of colonoscope type and $95 \%$ confidence interval $(\mathrm{Cl})$ were calculated using analysis of covariance (ANCOVA) and logistic regression with adjustment for stratification factors, age and prior abdomino-pelvic surgery. All statistical analyses were performed using IBM SPSS Statstiics version 26 (SPSS Inc, Chicago, Illinois, United States).

\section{Results}

\section{Enrollment and baseline characteristics}

A total of 220 patients were randomized to the small-caliber colonoscope group $(n=110)$ or standard colonoscope group $(n=$ 110) during the study period. A CONSORT flow diagram of the study patients is presented in $\mathbf{F i g}$. 2. No patient withdrew consent after randomization. In the small-caliber colonoscope group, colonoscopy was incomplete in one patient $(0.9 \%)$; she had an obstructive colon cancer and there was no switch to the standard colonoscope in that session because of severe bowel stricture. In the standard colonoscope group, colonoscopy was incomplete in eight patients (7.3\%); all of these patients had excessive looping because of floppy and redundant colons. After switching to the small-caliber colonoscope, cecal intubation was successful in all eight patients. A single patient in the small-caliber colonoscope group was given pentazocine with intravenous infusion as sedation during colonoscopy, because of severe pain due to bowel adhesion.

The demographic and clinical characterisitics of the smallcaliber colonoscope group and the standard colonoscope group were similar ( $\triangleright$ Table 1 ). The mean age (SD) was 62.7 years (13.3) among patients in the small-caliber colonoscope 
Enrollment $(\mathrm{n}=220)$

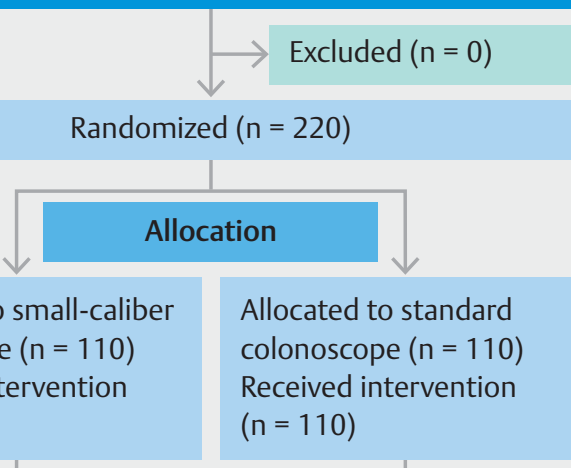

Follow up

Allocated to small-caliber colonoscope $(n=110)$ Received intervention $(n=110)$

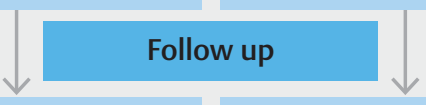

\section{Lost to follow up $(n=0) \quad$ Lost to follow up $(n=0)$}

\section{Analysis}

Intention-to-treat
analysis $(\mathbf{n}=\mathbf{1 1 0})$
- Complete colonoscopies
with 1st scope $(n=109)$
- Incomplete colonoscopy
due to obstructive colon
cancer $(n=1)$

Intention-to-treat analysis $(n=110)$

- Complete colonoscopies with 1st scope $(n=102)$

- Complete colonoscopies with 2nd scope $(n=8)$

Fig. 2 CONSORT flowchart of the study patients.

group and 63.1 years (12.6) among those in the standard colonoscope group. Sixty-two of 110 patients (56\%) in the smallcaliber colonoscope group and 59 of 110 patients (54\%) in the standard colonoscope group had prior abdomino-pelvic surgery.

\section{Primary and secondary outcomes}

The overall pain VAS score and the maximum pain VAS score were significantly lower in the small-caliber colonoscope group than in the standard colonoscope group (overall pain, mean [SD], 20.0 [21.6] vs. 32.4 [25.4], $P<0.0001$; maximum pain, mean [SD], 28.9 [27.0] vs. 47.2 [30.0], $P<0.0001$; $>$ Table 2). The cecal intubation rate with 1 st colonoscope was significantly superior in the small-caliber colonoscope group to the standard colonoscope group ( $99 \%$ vs. $93 \%, P=0.035$; $>$ Table 2). The withdrawal time in the small-caliber colonoscope group was significantly longer than that in the standard colonoscope group (small-caliber colonoscope vs. standard colonoscope: mean [SD], 13.1 [5.7] minutes vs. 11.4 [4.7] minutes, $P=$ 0.017; $>$ Table 2). The cecal intubation time and total procedure time did not differ significantly between the two groups (small-caliber colonoscope vs. standard colonoscope: cecal intubation time, mean [SD], 11.7 [9.0] minutes vs. 13.0 [8.5] minutes, $P=0.226$; total procedure time, mean [SD], 24.8 [10.0] minutes vs. 24.4 [10.2] minutes, $P=0.779$; $>$ Table 2). The adenoma detection rate did not differ significantly between the two groups (small-caliber colonoscope vs. standard colonoscope: $45 \%$ vs. $44 \%, P=0.892$; Table 2 ). The rate of abdominal compression used in the small-caliber colonoscope

- Table 1 Patient demographic and clinical characteristics.

\begin{tabular}{|c|c|c|c|}
\hline Factor & $\begin{array}{l}\text { Overall } \\
(n=220)\end{array}$ & $\begin{array}{l}\text { Small-caliber colonoscope } \\
(n=110)\end{array}$ & $\begin{array}{l}\text { Standard colonoscope } \\
(n=110)\end{array}$ \\
\hline Age, mean (SD), years & $62.9(12.9)$ & $62.7(13.3)$ & $63.1(12.6)$ \\
\hline Height (SD), cm & $154.2(6.0)$ & $154.4(6.2)$ & $154.0(5.8)$ \\
\hline Weight (SD), kg & $52.7(10.0)$ & $52.4(10.0)$ & $53.0(10.1)$ \\
\hline Waist (SD), cm & $79.4(10.8)$ & $78.8(10.5)$ & $80.0(11.1)$ \\
\hline $\mathrm{BMI}(\mathrm{SD}), \mathrm{kg} / \mathrm{m}^{2}$ & $22.2(4.0)$ & $22.0(3.8)$ & $22.4(4.1)$ \\
\hline \multicolumn{4}{|l|}{ Indication for colonoscopy } \\
\hline - Positive FIT, n (\%) & $75(34)$ & $34(31)$ & $41(37)$ \\
\hline - Screening, n (\%) & $45(20)$ & $27(25)$ & $18(17)$ \\
\hline - Surveillance, n (\%) & $39(17)$ & $18(16)$ & $21(19)$ \\
\hline - Hematochezia, n \%) & $17(8)$ & $7(6)$ & $10(9)$ \\
\hline - Others, n (\%) & $44(20)$ & $24(22)$ & $20(18)$ \\
\hline \multicolumn{4}{|l|}{ Other co-existed factors } \\
\hline - Prior abdomino-pelvic surgery, n (\%) & $121(55)$ & $62(56)$ & $59(54)$ \\
\hline - Sigmoid colon diverticulum, n (\%) & $33(15)$ & $13(12)$ & $21(19)$ \\
\hline - Fair/poor bowel preparation, n (\%) & $41(19)$ & $21(19)$ & $20(18)$ \\
\hline - Inexperienced endoscopist, n (\%) & $68(31)$ & $33(30)$ & $35(32)$ \\
\hline
\end{tabular}


- Table 2 Primary and secondary outcomes in the small-caliber group and standard colonoscope groups.

\begin{tabular}{|c|c|c|c|c|c|}
\hline Factors & $\begin{array}{l}\text { Small-caliber } \\
\text { colonoscope }\end{array}$ & $\begin{array}{l}\text { Standard } \\
\text { colonoscope }\end{array}$ & $P$ value & Difference $^{1}$ & $95 \% \mathrm{Cl}$ \\
\hline Overall VAS score, mean (SD) & $20.0(21.6)$ & $32.4(25.4)$ & $<0.0001^{2}$ & -12.4 & -18.7 to -6.1 \\
\hline Maximum VAS score, mean (SD) & $28.9(27.0)$ & $47.2(30.0)$ & $<0.0001^{2}$ & -18.3 & -25.9 to -10.7 \\
\hline Cecal intubation rate, $\mathrm{n}(\%)$ & 109 (99) & $102(93)$ & $0.035^{3}$ & 0.1 & 0.009 to 0.11 \\
\hline Cecal intubation time, median (SD), minutes & $11.7(9.0)$ & $13.0(8.5)$ & $0.266^{2}$ & -1.3 & -3.7 to 1.0 \\
\hline Withdrawal time, median (SD), minutes & 13.1(5.7) & $11.4(4.7)$ & $0.017^{2}$ & 1.7 & 0.31 to 3.09 \\
\hline Total procedure time, median (SD), minutes & $24.8(10.0)$ & $24.4(10.2)$ & $0.779^{3}$ & 0.4 & -2.30 to 3.07 \\
\hline Adnoma detection, $\mathrm{n}(\%)$ & $49(45)$ & $48(44)$ & $0.892^{3}$ & 0.0 & -0.12 to 0.14 \\
\hline Use of abdominal compression, $\mathrm{n}(\%)$ & $56(51)$ & $73(66)$ & $0.020^{3}$ & -0.2 & 0.15 to 0.16 \\
\hline Patients' acceptance for colonoscopy, n (\%) & $108(98)$ & $96(87)$ & $0.003^{3}$ & 0.1 & 0.07 to 0.15 \\
\hline Adverse event, n (\%) & $0(0)$ & $0(0)$ & $>0.999^{3}$ & 0.0 & - \\
\hline \multicolumn{6}{|c|}{$\begin{array}{l}\mathrm{Cl} \text {, confidence interval; VAS, visual analogue scale; SD, standard deviation. } \\
{ }^{1} \text { Difference of mean or proportion between the two groups. } \\
{ }^{2} \text { Student's t-test. } \\
{ }^{3} \text { Chi-square test or Fisher's extract test }\end{array}$} \\
\hline
\end{tabular}

- Table 3 Comparison of colonoscope type and patient pain using analysis of covariance.

\begin{tabular}{|c|c|c|c|c|c|}
\hline \multirow[t]{2}{*}{ Group } & \multicolumn{2}{|l|}{ Overall VAS score } & \multicolumn{3}{|l|}{ Difference } \\
\hline & Adjusted mean VAS score ${ }^{1}$ & $95 \% \mathrm{Cl}$ & Adjusted mean VAS score & $95 \% \mathrm{Cl}$ & $P$ value \\
\hline Standard colonoscope & 32.3 & 27.8 to 36.7 & \multirow[t]{2}{*}{-12.5} & \multirow[t]{2}{*}{-18.8 to -6.2} & \multirow[t]{2}{*}{$<0.0001$} \\
\hline Small-caliber colonoscope & 19.7 & 15.3 to 24.2 & & & \\
\hline
\end{tabular}

- Table 4 Logistric regression analysis of effects of colonoscope type on acceptance rate for future unsedated colonoscopy.

\begin{tabular}{|l|l|l|l|l|l|}
\hline Group & Odds ratio & $\mathbf{9 5 \%} \mathbf{C l}$ & P value & Adjusted odds ratio & $\mathbf{9 5 \%} \mathbf{C l}$ \\
\hline Standard colonoscope & 1 & & 1 & $\mathbf{P}$ value \\
\hline Small-caliber colonoscope & 7.88 & 1.75 to 35.54 & 0.007 & 8.21 & 1.80 to 37.29 \\
\hline $\begin{array}{l}\text { Cl, confidence interval. } \\
\text { 1 Odds ratio was adjusted with age and prior abdomino-pelvic surgery on the basis of logistic regression. }\end{array}$ & 0.006 \\
\hline
\end{tabular}

group was significantly lower than that in the standard colonoscope group ( $51 \%$ vs. $66 \%, P=0.02$; $>$ Table 2 ). In addition, the rate of patients' acceptance of unsedated colonoscopy in the future was higher in the small-caliber colonoscope group than the standard colonoscope group ( $98 \%$ vs. $87 \%, P=0.003$; $>$ Table 2). No AEs were observed in either group.

\section{ANCOVA and logistic regression assessing the group differences}

ANCOVA assessment of the group difference for overall pain VAS score, after adjustment for age and prior abdomino-pelvic surgery, showed that the mean overall pain VAS score was significantly lower in the small-caliber colonoscope group than the standard colonoscope group (19.7 vs. $32.3, P<0.0001$; - Table 3). The odds ratio, adjusted with age and prior abdomino-pelvic surgery, for the acceptance rate of unsedated colonoscopy with the small-caliber colonoscope as compared with the standard colonoscope was $8.21(P=0.006$, 95\% Cl $1.80-$ 37.29; Table 4).

\section{Discussion}

This RCT showed that colonoscopy using the small-caliber colonoscope for female patients can significantly reduce their pain during unsedated colonoscopy and improve their acceptance of unsedated colonoscopy in the future. The cecal intubation rate 
with the small-caliber colonoscope was significantly superior to that with the standard colonoscope. The cecal intubation time, total procedure time, and adenoma detection rate did not differ between the two groups.

A meta-analysis has shown that small-caliber colonoscopes are superior to standard colonoscopes with respect to cecal intubation rates and pain scores, and it also showed that the cecum intubation time and adenoma detection rate did not differ significantly between the two colonoscopes [22]. These findings match the results from our study.

Among the studies focusing on women, Nemoto et al. showed that median overall pain scores were significantly lower using a small-caliber colonoscope, with an outer diameter of $7.0 \mathrm{~mm}$, than for a pediatric colonoscope, with an outer diameter of $11.1 \mathrm{~mm}$, in unsedated women of advanced age [23]. Sato et al. showed in their subgroup analysis that the median overall pain and median maximum pain scores were significantly lower in the small-caliber colonoscope (PCF-PQ260 L) group than in the standard colonoscope, with an outer diameter of $12.2 \mathrm{~mm}$, group in sedated women [18]. Our study produced a similar result to these previous studies focusing on women. The decrease in patient pain in our study is attributable to the small caliber of the colonoscope, as in previous reports [9, 16-19]. In addition, we believe that the passive bending of the PCF-PQ260 $L$ provided a supplementary benefit, especially for passage of the colonoscope through the sharp flexure.

Our study also has several strengths to reach a convincing and generalizable conclusion. First, this study was analyzed with intention-to-treat for all enrolled patients. Thus, the result from our study reflects the real world of clinical practice. Second, this study illustrates the value of the small-caliber colonoscope for reducing patient pain, with a larger number of participating endoscopists than previous studies. Thus, we believe that the small-caliber colonoscope is suitable for use by various endoscopists, with the consequence of improving their performance. Third, ANCOVA and logistic regression were used to adjust for stratification factors, age and prior abdomino-pelvic surgery, and they demonstrated that the small-caliber colonoscope group was superior with respect to reducing the patient's pain and increasing the acceptance rate of unsedated colonoscopy in the future.

In general, small-caliber colonoscopes have a disadvantage in that their small-caliber and flexible natures result in excessive looping on insertion into the cecum, especially in patients with a redundant colon $[24,25]$. This can result in small-caliber colonoscopes being unable to be advanced towards the cecum in the proximal colon and requiring more frequent abdominal compressions. However, the result of our study demonstrated that the requirement for abdominal compression to advance the colonoscope was lower in the small-caliber colonoscope group than the standard colonoscope group. This result might be associated with the design of the PCF-PQ260L, which is equipped with a high force transmission.

Several limitations of our study should be addressed. First, the endoscopist and medical assistant were not blinded; thus, the endoscopists knew which colonoscope was being used during colonoscopy and investigator bias could be not excluded.
Second, the generalization of our findings may be limited because it was a single-center study.

\section{Conclusions}

In conclusion, this RCT focusing on women confirmed that the small-caliber colonoscope PCF-PQ260 L could significantly reduce pain during unsedated colonoscopy and improve the rate of patient acceptance of unsedated colonoscopy in the future. In reducing the use of sedation, providing less painful colonoscopy, and promoting acceptance of colonoscopy for CRC screening and surveillance, the PCF-PQ260 L is a valuable tool in colonoscopy for women.

\section{Acknowledgements}

The authors thank Hiroyuki Inoue, Takashi Kitade, Syunsuke Tano, Takashi Sakuno, Tetsuro Harada, Hiroshi Miura, Hiroaki Okuse, and the other medical staff members of the Department of Endoscopy, Mie University Hospital for their contribution to this work.

\section{Competing interests}

The authors declare that they have no conflict of interest.

\section{References}

[1] Bray F, Ferlay J, Soerjomataram I et al. Global cancer statistics 2018: GLOBOCAN estimates of incidence and mortality worldwide for 36 cancers in 185 countries. CA Cancer J Clin 2018; 68: 394-424

[2] Zauber AG, Winawer SJ, O'Brien M] et al. Colonoscopic polypectomy and long-term prevention of colorectal-cancer deaths. N Engl J Med 2012; 366: 687-696

[3] Ghevariya V, Duddempudi S, Ghevariya N et al. Barriers to screening colonoscopy in an urban population: a study to help focus further efforts to attain full compliance. Int J Colorectal Dis 2013; 28: 14971503

[4] Luo H, Zhang L, Liu X et al. Water exchange enhanced cecal intubation in potentially difficult colonoscopy. Unsedated patients with prior abdominal or pelvic surgery: a prospective, randomized, controlled trial. Gastrointest Endosc 2013; 77: 767-773

[5] Morgan J, Thomas K, Lee-Robichaud H et al. Transparent cap colonoscopy versus standard colonoscopy to improve caecal intubation. Cochrane Database Syst Rev 2012; 12: Cd008211

[6] Mark-Christensen A, Brandsborg S, Iversen LH. Magnetic endoscopic imaging as an adjuvant to elective colonoscopy: a systematic review and meta-analysis of randomized controlled trials. Endoscopy 2015; 47: $251-261$

[7] Othman MO, Bradley AG, Choudhary A et al. Variable stiffness colonoscope versus regular adult colonoscope: meta-analysis of randomized controlled trials. Endoscopy 2009; 41: 17-24

[8] Park CH, Lee WS, Joo YE et al. Sedation-free colonoscopy using an upper endoscope is tolerable and effective in patients with low body mass index: a prospective randomized study. Am J Gastroenterol 2006; 101: 2504-2510

[9] Luo DJ, Hui A], Yan KK et al. A randomized comparison of ultrathin and standard colonoscope in cecal intubation rate and patient tolerance. Gastrointest Endosc 2012; 75: 484-490 
[10] Saunders BP, Fukumoto M, Halligan S et al. Why is colonoscopy more difficult in wo men? Gastrointest Endosc 1996; 43: 124-126

[11] Kim WH, Cho YJ, Park JY et al. Factors affecting insertion time and patient discomfort during colonoscopy. Gastrointest Endosc 2000; 52: 600-605

[12] Shah SG, Brooker JC, Thapar C et al. Patient pain during colonoscopy: an analysis using real-time magnetic endoscope imaging. Endoscopy 2002; 34: 435-440

[13] Bugajski M, Wieszczy P, Hoff G et al. Modifiable factors associated with patient-reported pain during and after screening colonoscopy. Gut 2018; 67: 1958-1964

[14] Predmore Z, Nie X, Main R et al. Anesthesia service use during outpatient gastroenterology procedures continued to increase from 2010 to 2013 and potentially discretionary spending remained high. Am J Gastroenterol 2017; 112: 297-302

[15] Wernli KJ, Brenner AT, Rutter CM et al. Risks associated with anesthesia services during colonoscopy. Gastroenterology 2016; 150: 888894; quiz e818

[16] Tox U, Schumacher B, Toermer T et al. Propofol sedation for colonoscopy with a new ultrathin or a standard endoscope: a prospective randomized controlled study. Endoscopy 2013; 45: 439-444

[17] Garborg KK, Loberg M, Matre J et al. Reduced pain during screening colonoscopy with an ultrathin colonoscope: a randomized controlled trial. Endoscopy 2012; 44: 740-746
[18] Sato K, Ito S, Shigiyama F et al. A prospective randomized study on the benefits of a new small-caliber colonoscope. Endoscopy 2012; 44: 746-753

[19] Sato K, Ito S, Kitagawa T et al. A prospective randomized study of the use of an ultrathin colonoscope versus a pediatric colonoscope in sedation-optional colonoscopy. Surg Endosc 2017; 31: 5150-5158

[20] Saito Y, Kimura H. Responsive insertion technology. Dig Endosc 2011; 23: (Suppl. 01): 164-167

[21] Kastenberg D, Bertiger G, Brogadir S. Bowel preparation quality scales for colonoscopy. World J Gastroenterol 2018; 24: 2833-2843

[22] Sofi AA, Nawras A, Khan MA et al. Meta-analysis of the performance of ultrathin vs. standard colonoscopes. Endoscopy 2017; 49: 351-358

[23] Nemoto D, Utano K, Endo S et al. Ultrathin versus pediatric instruments for colonoscopy in older female patients: A randomized trial. Dig Endosc 2017; 29: 168-174

[24] Marshall JB, Perez RA, Madsen RW. Usefulness of a pediatric colonoscope for routine colonoscopy in women who have undergone hysterectomy. Gastrointest Endosc 2002; 55: 838-841

[25] Saifuddin T, Trivedi M, King PD et al. Usefulness of a pediatric colonoscope for colonoscopy in adults. Gastrointest Endosc 2000; 51: 314317 